This document is the accepted manuscript version of the following article:

Schwiedrzik, J., Raghavan, R., Bürki, A., LeNader, V., Wolfram, U., Michler, J., \& Zysset, P. (2014). In situ micropillar compression reveals superior strength and ductility but an absence of damage in lamellar bone. Nature Materials, 13, 740-747. https://doi.org/10.1038/nmat3959

\title{
In situ micropillar compression reveals superior strength and ductility but an absence of damage in lamellar bone
}

J. J. Schwiedrzik, R. Raghavan, A. Bürki, V. LeNader, U. Wolfram, J. Michler, P.K. Zysset

Final Author Version

Nature Materials, 13, 740-747, 2014

DOI: $10.1038 / \mathrm{nmat} 3959$

Weblink: http://www.nature.com/nmat/journal/v13/n7/full/nmat3959.html 


\section{In situ micropillar compression of lamellar bone reveals superior strength and ductility but no damage}

Jakob Schwiedrzik ${ }^{\mathrm{a}, 1}$, Rejin Raghavan ${ }^{\mathrm{b}, 2}$, Alexander Bürki ${ }^{1}$, Victor LeNader $^{2}$, Uwe Wolfram ${ }^{1}$, Johann Michler ${ }^{c, 2}$, and Philippe Zysset ${ }^{c, 1}$

${ }^{1}$ Institute for Surgical Technology and Biomechanics, University of Bern, Stauffacherstr. 78, CH-3014 Bern, Switzerland ${ }^{2}$ EMPA, Swiss Federal Laboratories for Material Science and

Technology, Laboratory of Mechanics of Materials and Nanostructures, Feuerwerkerstr. 39, CH-3602, Switzerland

March 24, 2014

${ }^{a}$ Corresponding author. Email: jakob.schwiedrzik@istb.unibe.ch

b Current address: Max-Planck-Institut für Eisenforschung, Structure and Nano/Micromechanics of Materials, Max-Planck.Str. 1, D-40237 Düsseldorf, Germany

${ }^{\mathrm{c}} \mathrm{JM}$ and $\mathrm{PZ}$ share senior authorship for this article. 
Aging societies suffer from an increasing incidence of bone fractures. Bone strength depends on the amount of mineral measured by clinical densitometry, but also on the micromechanical properties of the bone hierarchical organization. Here, we investigated the mechanical response under monotonic and cyclic compression of both single osteonal lamellae and macroscopic samples containing numerous osteons. Micropillar compression tests in a scanning electron microscope, microindentation and macroscopic compression tests were performed on dry ovine bone to identify elastic modulus, yield stress, plastic deformation, damage accumulation and failure mechanisms. We found that isolated lamellae exhibit a plastic behavior with higher yield stress and ductility but no damage. In agreement with a proposed rheological model, these experiments illustrate a transition from a ductile mechanical behavior of bone at the microscale to a quasi-brittle response driven by the growth of cracks along interfaces or in the vicinity of pores at the macroscale.

Bone is a hierarchical composite material featuring a cell-seeded mineralized collagen matrix. It is designed for mechanical support, metabolizing minerals and storing bone marrow $[1,2]$ and mostly loaded in compression in everyday activities [3]. Mineralized collagen fibrils surrounded by extrafibrillar mineral particles $\underline{[4,3,5]}$ combine into fibril arrays. In lamellar bone, parallel fibril arrays form lamellae in a rotated plywood pattern $[6,7]$. Osteocytes and their processes inhabit the lacuno-canalicular network which makes up for about $1 \%$ of whole bone porosity [8]. Human compact bone consists of lamellae arranged concentrically around blood vessels forming osteons with a porosity of around $6 \%$ [1]. In large, fast growing animals an alternative tissue type, the so called fibrolamellar bone, is laid out first and converted to osteonal bone through a remodeling process [3].

Biomechanical testing of bone on the macroscale has been performed for more than a century. However, many challenges remain, mostly due to spatial, inter-subject, age, and disease variation of mechanical properties $[9,10]$. Analysis of bone as a hierarchical composite is an important field in biomechanics $[1,11,12]$ trying to understand and predict whole bone properties more accurately by considering its mechanical behavior on the lower length scales. However, so far it suffers from a lack of direct measurements of postyield properties on the microscale.

There is evidence on the continuum level that bone shows two simultaneous mechanisms of energy dissipation on the macroscale when loaded quasi-statically past the yield point: inelastic deformation of the material and damage (i.e. reduction of stiffness through the formation of microcracks) $[13,14,15]$. Negative fibril strains following macroscopic tensile yielding found by Gupta et al. [16] hint at damage as a dissipation mechanism on a lower length scale. However, these findings on the microscale 
were reported following macroscopic tests and are therefore influenced by the microstructure of the tested specimens. Therefore, it is necessary to perform tests directly on the microscale in order to decouple material and structural effects.

Indentation is a mechanical testing technique in which a tip with known geometry is pressed into a flat sample surface and force and tip displacement are recorded. The pioneering work of Oliver and Pharr [17] allows us to extract elastic properties from the unloading part of the indentation curve. Indentation in bone with depths up to $1 \mu \mathrm{m}$ mainly aims at characterizing the mechanical properties on the lamellar $(3-7 \mu \mathrm{m})$ level $[18,19]$. Finite element simulations of indentations using coupled plasticity and damage models have shown that damage may explain some of the experimental findings for bone like a reduced unloading stiffness $[20,21]$ and that strength on the microlevel seems to be similar to the macroscopic values. However, it is very difficult to uniquely interpret such experimental data in materials featuring dissipative processes, as indentations in materials with different behaviors can result in very similar curves $[22]$. Therefore, independent experiments allowing a straightforward interpretation are necessary in order to assess the deformation mechanisms and postyield behavior of bone at the microscale.

Such an experimental setup for micromechanical testing is micropillar compression. Micron sized pillars are produced by erosion of material using a focused ion beam (FIB) and used to extract mechanical properties of all classes of materials [23, 24]. Due to the uniaxial loading conditions as opposed to the complex stress states during indentations, the setup allows a straightforward interpretation of the data. Also, this technique is ideal for studying the effects of size in quasi-brittle materials [25] in terms of determining the postyield properties and deformation mechanisms by circumventing premature fracture $[23,24,26,27]$.

For brittle materials like ceramics failure is associated with the growth of cracks originating from pores, surface scratches or other preexisting defects. When testing smaller specimens, the probability that a defect of a certain critical size is present in the material decreases, which leads to an increased failure stress [28]. When reaching very small sample sizes, no defects of critical size may be present and alternative dissipative processes like dislocation based plasticity dominate [24]. In bone, the hierarchical structure leads to a macroscopically quasi-brittle behaviour where plasticity and cracking both play a significant role and the associated scaling law remains to be investigated.

The aim of this study was therefore to investigate: what is the anisotropic uniaxial compressive strength of lamellar bone at the microscale; what are the dominating deformation and failure mechanisms under compression in the postyield regime on the microscale; and how do these properties compare to the macroscopic response? Based on the reviewed literature $[29,16,21$, 
30, 20], we intend to test two hypotheses: 1 . The compressive strength of bone is similar on the lamellar and macroscopic level. 2. Under compression, bone deforms inelastically in a quasi-brittle fashion by formation of cracks on several length scales, which lead to a reduction of stiffness at both levels.

In order to test these hypotheses, in situ micropillar compression tests using monotonic and cyclic loading protocols and microindentation tests were performed on dry ovine osteonal bone in axial and transverse direction and compared to macroscopic uniaxial compression tests using the same sample geometry and loading protocol.

Micropillar diameter and aspect ratio before testing were determined by scanning electron microscopy (SEM) to be $5.21 \pm 0.14 \mu \mathrm{m}$ (mean \pm standard deviation) and $2.13 \pm 0.10$. Based on the in situ observation of the micropillar compression tests in a SEM and high resolution SEM (HRSEM) pictures taken after testing (Fig. 2), the failure modes were classified into three groups: shearing, mushrooming, and axial splitting. Under axial compression, $55 \%$ of the pillars failed by development of shear planes, $25 \%$ by mushrooming, i.e. failure on the top of the pillar leading to localized cracking and delamination, and $15 \%$ by an axial split. One pillar failed by compression of a relatively large pore and was removed from the study. All of the pillars oriented in transverse direction failed by shearing. The true stress-strain curves resulting from the analysis of the quasi-static micropillar compression as well as normalized postyield behavior may be seen in Fig. 1. For the micropillars, yield stress was found to be $0.49 \pm 0.1 \mathrm{GPa}$ in axial $(N=19)$ and $0.30 \pm 0.02 \mathrm{GPa}$ in transverse direction $(N=20)$, strength was $0.75 \pm 0.06 \mathrm{GPa}$ and $0.59 \pm 0.04 \mathrm{GPa}$, respectively. The postyield behavior depended on the failure mode. Strain hardening/softening behavior was observed in the case of mushrooming and splitting, continuous hardening until failure in the case of shearing (Fig. 1 bottom). A linear regression model showed that testing direction was a significant parameter $\left(p=9 \times 10^{-10}\right)$ and could explain $71.9 \%$ of the variation of strength and $63.8 \%$ of the yield stresses, while failure mode was not a significant parameter $(p=0.1)$. When assessing the pillars that showed shear failure only, direction alone accounts for $84 \%$ of the variation of strength and $74.8 \%$ of the yield stresses. For 22 out of the 31 pillars that failed by localization in a single shear plane, the inclination angle could be measured and was found to have a mean of $46.63 \pm 8.94^{\circ}$, with no significant difference between axial and transverse directions $(p=0.63)$. For this data subset, the critical shear stress along the plane of failure was computed and analyzed using analysis of variance (ANOVA). It was found that only loading direction was a significant parameter $\left(p=2 \times 10^{-6}\right)$ with critical shear stresses of $0.356 \pm 0.028 \mathrm{GPa}$ in axial and $0.280 \pm 0.026 \mathrm{GPa}$ in the transverse direction. HRSEM micrographs of the slip planes of micropillars failed by shearing revealed three main toughening mechanisms of bone: isolated fibril bridging as well as ligament bridging and crack deflection. Fibril bridging was almost exclusively 
observed in axial micropillars (Fig. 5).

The apparent modulus measured during the cyclic micropillar compression (Fig. 4) was found to be $31.16 \pm 6.46 \mathrm{GPa}$ in axial $(N=5)$ and $16.5 \pm 1.50 \mathrm{GPa}$ in transverse direction $(N=5)$. Analysis of the normalized apparent modulus as a function of plastic strain showed that the stiffness is not reduced after overloading of the sample to up to $8 \%$ plastic strain (Fig. 4). A linear regression showed no significant change of normalized apparent modulus as a function of plastic strain $(p=0.188$, RMS error $=0.032, N=50$ ) for the pillars that failed by development of a slip plane. When fitting only the axial pillars that failed in shear, a nonsignificant slope was found $(p=0.026)$ predicting a $5 \%$ modulus reduction at $8 \%$ of plastic strain, which is 20 times smaller than for the macroscopic data reported later in this manuscript.

Microindentation showed also a clear anisotropy of mechanical properties. The measured values for indentation modulus, hardness and work in wet and dry state may be seen in Tab. 1 and are in accordance with values for dry human osteonal bone of Reisinger et al. [31], who reported indentation moduli of $27.6 \pm 3.3 \mathrm{GPa}$ and $20.5 \pm 1.9 \mathrm{GPa}$ in the axial and transverse directions. A mean stiffness tensor was fitted to the average dry indentation moduli using the methodology of Franzoso and Zysset [32]. The resulting predicted apparent moduli were found to be $28.97 \mathrm{GPa}$ in axial and 16.07 GPa in transverse direction, respectively.

Visual inspection of 2 cross sections in 15 of the macroscopic samples showed that $\sim 50 \%$ of them were entirely secondary osteonal, the others showed a mixture of fibrolamellar and osteonal bone. During the macroscopic cyclic tests apparent moduli of $25.74 \pm 3.71 \mathrm{GPa}$ and strength of $0.314 \pm 0.043 \mathrm{GPa}$ were measured $(N=26)$. The stress-strain curves and normalized modulus evolution as a function of plastic strain are shown in Fig. 4. The macroscopic samples showed a significantly lower strength than the micropillars $\left(p<2 \times 10^{-16}\right)$ and a much more brittle behavior. The samples failed mostly at less than $1.2 \%$ of plastic strain, which is 6 times less than on the microscale and consistent with the literature [33]. Ductility of the osteonal samples was not higher than for the rest of the group. It was found that modulus was significantly reduced as a function of plastic strain (Fig. 3, $p=2.4 \times 10^{-10}$, adj. $R^{2}=0.3, N=116$ ), which is consistent with reports for tension $[10,15]$. A one dimensional rheological model based on elasto-plasticity with failure (Fig. 3, Supplementary Fig. 8 and 9) representing bone at the microscale was fed with the means and standard deviations of the elastic modulus, yield stress and maximal plastic strain measured in the micropillar experiments (Supplementary Tab. 1). Using an appropriate plastic hardening law, the resulting stress-strain curves match the experimental data in both axial and transverse directions very well (Fig. 4) and no stiffness reduction is observed below $8 \%$ of plastic strain. In order to account for the existing cracks and defects between bone structural units in the 
macroscopic samples, the rheological model was fed with a homogenous distribution of yield stress and maximum plastic strain bounded by microscale properties (Supplementary Fig. 10). With this single assumption that is in line with the argument made by Krajcinovic [34] in a damage model for bone under tension, the model predicted a strength of $0.3 \mathrm{GPa}$ and a modulus reduction of $11.5 \%$ at a plastic strain of $1.2 \%$. Also, the predicted stress-strain curve matched the experimental data very well (Fig. 4).

The microindentation measurements were in very good agreement with the results reported by Reisinger et al. [31] for dry human osteonal bone. This confirms that ovine bone is an acceptable model for human tissue at the lamellar level as indicated by $[35,36]$. The macroscopic strength measured in this study was slightly higher than the $0.272 \pm 0.003 \mathrm{GPa}$ reported by Reilly and Burstein [37] for Haversian bovine bone. This is in line with the fact that Haversian systems tend to reduce the strength of primary bone [3]. The depth of the microindentations and the dimensions of the micropillars were chosen to be consistent with the average lamellar thickness of 3-7 $\mathrm{\mu m}$. Consequently, the apparent modulus of the cyclic tests after correction using the Sneddon approach [38] corresponded very well to the apparent moduli predicted from the microindentation data. Also, the high consistency of the micropillar compressions demonstrated the homogeneity of ovine bone at this length scale in the absence of interfaces such as cement lines. The macroscopic tests were designed with the same aspect ratio and strain rate as the micropillar tests, which allowed to do a direct comparison of the two length scales.

Three distinct failure modes could be identified on the microlevel from the in situ observation and post-failure HRSEM micrographs: shearing, mushrooming, and axial splitting. The dominating failure mode was shearing with almost $80 \%$ of the samples showing a highly ductile behavior with continuous hardening until failure by localization in a slip plane. This observed behavior is consistent with oblique or cross-hatched slip lines reported for compressive yielding of compact bone on the macroscale [33, 3]. A minority of the axial micropillars failed by mushrooming associated with boundary effects such as indenter-sample friction or axial splitting, which is a brittle failure mode [27].

The observed postyield behavior of the sheared micropillars was anisotropic: axial pillars had a higher yield to ultimate stress ratio and reached their strength at lower strains than transverse pillars, but failed less rapidly after the maximum stress had been passed. HRSEM pictures revealed failure planes with ligament bridging, crack deflection, and, almost exclusively for axial pillars, isolated fibril bridging. These phenomena act as toughening mechanisms and are consistent with similar findings of other groups $[39,40,41,42]$. Measurement of the inclination angles showed that on average the failure occurred in the plane of maximum shear stress. The critical shear stress in the slip plane was significantly anisotropic. The direction- 
dependence of the observed postyield behavior and the critical shear stress hints at a difference in the failure mechanism and may be explained by a nonlinear rotated plywood model: For the axial pillars, the majority of the fibrils are oriented at small angles to the loading direction. The observed slip planes are a result of a combined fibril and extra-fibrillar mineral failure. In this case, isolated fibrils that have not yet failed act as a toughening mechanism leading to an increased post strength ductility. For the transverse micropillars, most of the fibrils are oriented at angles close to $90^{\circ}$ to the loading direction. Therefore, one orientation exists for which the number of fibers going across the plane is minimal. In this case, a slip plane may form by failure of the extra-fibrillar mineral only, which explains the absence of fibrils spanning the gap and the rapid failure once strength had been passed in most of the transverse micropillars (Fig. 5). It was found that an elastoplastic model with failure (Fig. 3) is able to represent the microscale data very accurately (Fig. 4). The observed plasticity could be explained by different nanoscale mechanisms, e.g. nanogranular friction between mineral particles [43] or dissipation at the interface between mineral platelets and the organic phase $[44,45]$.

When comparing the two length scales, only a small difference in stiffness of $\sim 10 \%$ was found. When applying an equal strain model, this difference could be explained by a porosity of $10 \%$, which is in the range of the lacunarcanalicular and Haversian porosity [1]. On the other hand, the strength and failure behavior differed strongly at the two length scales. The response on the microlevel was highly ductile with maximum plastic strains in the order of $8 \%$, while the dry macroscopic samples failed mostly in a quasi-brittle fashion with plastic strains below $1.2 \%$. The measured strength on the microscale was higher by a factor of 2.4 than the macroscopic tests. When applying the quadratic relationship between strength and porosity proposed by Carter and Hayes [46], a change in strength by a factor of only 1.23 would be expected, therefore the first hypothesis that ultimate stresses are similar on the micro- and macroscale was falsified. The unaccounted difference in strength and ductility is attributed to a size effect, more specifically to the existing cracks and defects located between osteons [47], and is in line with the scaling theory of quasi-brittle failure [25]. The yield stress and ultimate plastic strain associated with the growth of pre-existing cracks near interfaces $[48,49]$ or pores [50] on the macroscale are substantially lower than the ones necessary to initiate and propagate a new crack through a micropillar made of a single osteonal lamella (Supplementary Fig. 7). The proposed rheological model (Fig. 3) illustrates this difference very well as it was found to be in excellent agreement with the experimental data at both length scales (Fig. 4). The model is thus able to build a bridge between the newly observed ductile behaviour on the lamellar level and the well-documented quasi-brittle behaviour of bone on the macroscale characterized by a reduction of stiffness that increases with plastic deformation 
[51]. Similar size effects are known for other material like ceramics [28], which show brittle failure due to crack growth near preexisting defects that are homogeneously distributed in the material. In the case of bone, these defects are concentrated in interstitial tissue and near cement lines [47], which causes the macroscopic response to be a combination of plastic deformation and cracking, while isolated osteonal lamellae show plasticity only.

Due to the setup of the in situ indenter in a conventional SEM and the preparation of the micropillars using a FIB, the tests were performed in a vacuum environment. Therefore, the specimens had to be dried before testing, which affects the mechanical properties of the organic phase and the surface chemistry of the mineral platelets. Raman spectroscopy confirmed that the composition was not significantly different from hydrated human samples reported in the literature (Supplementary Fig. 1 and 2). To assess the mechanical influence of hydration on the microscale, microindentations were performed in wet and dry condition. Indentation modulus increased by $20 \%$ in the axial direction, hardness and work by as much as $65 \%$ after drying. Hardness has been shown to scale with yield strength [52], which implies that stiffness and strength of hydrated micropillars would be reduced as compared to the dry setup. This is consistent with findings on the macroscale [53] . However, the ratio of plastic to total indentation work did not change significantly $(p=0.23)$, which is consistent with data for human bone [54] and suggests that the underlying dissipative mechanism remains the same after dehydration. Macroscopically, it has been reported that toughness is reduced by dehydration [53]. Nevertheless, the micropillars showed a highly ductile behavior up to failure. Therefore it might be argued that reduced toughness after drying is caused by its influence on interfaces and preexisting defects rather than on the bone matrix itself. This effect needs further investigation.

The yield and ultimate properties on the lamellar level reported in this study were also significantly higher than values reported in previous studies based on microindentation [30]. However, these studies had to rely on assumptions regarding the nonlinear material behavior and inverse methods to back-calculate material properties, while the data of the micropillar compression tests may be interpreted in a straightforward manner due to the uniaxial stress state. Finally, previous indentation studies suggested that a damage mechanism at the ultrastructural level might be responsible for some of the characteristics of indentation experiments in bone such as a reduced unloading stiffness $[20,21]$. However, most of the monotonic tests did not show the strain softening behavior that is characteristic of a mechanical damage process. Additionally, unlike those performed on the macroscale, the cyclic micropillar compression tests did not show a significant reduction of apparent modulus following overloading of the sample. This is an indication that no diffuse cracks were opening inside the micropillars until failure and falsifies the second hypothesis that bone deforms inelastically by 
formation of microcracks reducing its stiffness at both length scales.

This study showed that unlike the consistent elastic properties, the postyield properties and failure mechanisms of bone under compression differ significantly at the two length scales. Isolated bone lamellae show a high strength and ductility but no damage and fail mostly by development of shear planes, while the response of macroscopic specimens containing numerous osteons is quasi-brittle with low strength and ductility, substantial damage and longitudinal cracks. The data supports the thesis that, under compressive loading, multiple slip planes emerging at the weak interfaces or in the vicinity of pores in the interstitial bone coalesce into microcracks, the statistical distribution and growth of which lead to a quasi-brittle failure at the macroscopic level. These findings highlight the importance of studying interface properties of cement lines and the stress concentration effect of pores in the interstitial tissue more closely. Also, they remain to be extended to human lamellar bone tissue, physiological testing conditions and further loading modes. Deciphering the micromechanical behavior of lamellar bone and its evolution with age, disease and treatment will help preventing bone fractures in the elderly.

\section{Methods}

For a more thorough description of the materials and methods, the reader is referred to the supplementary material. Sheep tibiae were acquired from a local butcher shop. Ovine bone is mostly primary, features less porosity and a more homogeneous but similar mineralization compared to human tissue [35, 36], making it an attractive model for human bone on the microlevel. Axial and transverse specimens were cut from the diaphyses with a diamond-coated band saw (Exact, Germany), embedded in, but not infiltrated by, polymethylmethacrylate (PMMA), air-dried [54] for more than $48 \mathrm{~h}$, and subsequently ultramilled (Polycut E, Reichert-Jung, Germany). Raman spectroscopy was performed on a transverse control sample showing that the chemical composition of the sample was in the expected range (Supplementary Fig. 1 and 2).

Microindentations were performed in osteonal regions in dry and wet condition with a Ultra Nano Hardness Tester (UNHT, CSM Instruments, Switzerland). A trapezoidal protocol in load control up to a maximum depth of $1 \mu \mathrm{m}$ with a loading rate of $100 \mathrm{mN} / \mathrm{min}$, holding time of $30 \mathrm{~s}$ [54] and unloading rate of $400 \mathrm{mN} / \mathrm{min}$ was chosen to minimize the effects of creep. Indentation modulus and hardness, elastic and total work were extracted using standard methods [17, 31, 54]. A transversely isotropic stiffness tensor was fitted to the indentation moduli using the method of Franzoso and Zysset [32] to obtain apparent moduli.

After indentation, the samples were extracted from the embedding and 
glued on SEM stubs using silver adhesive. A $50 \mathrm{~nm}$ thick Au-Pd film was sputtered on the specimens to minimize drift due to charging. A dual beam FIB workstation (Tescan Lyra, Czech Republic) operated at $30 \mathrm{kV}$ was used to machine micropillars of $5 \mu \mathrm{m}$ diameter and aspect ratio of 2 in osteonal regions, and to section them after testing. Micropillars were aligned with the longitudinal and circumferential directions of the respective osteons. Circular trenches of $50 \mu \mathrm{m}$ diameter were machined with $6000 \mathrm{pA}$ to obtain $10 \mu \mathrm{m}$ diameter posts, which were milled down to $6 \mu \mathrm{m}$ with $1000 \mathrm{pA}$ and polished to $5 \mu \mathrm{m}$ with $300 \mathrm{pA}$ (Supplementary Fig. 3). Use of FIB can lead to damage of the material and Gallium implantation, which may affect the measurements. Monte Carlo simulations using the software SRIM [55] (incidence angle $3^{\circ}, 30 \mathrm{keV} \mathrm{Ga}{ }^{+}$ions, bone composition ICRU-119 from compound library) showed that damage is confined to $25 \mathrm{~nm}$ (Supplementary Fig. 4) and Gallium implantation to $15 \mathrm{~nm}$ within the surface and may be neglected, which is consistent with studies reporting no considerable FIB induced damage for mineralized tissues [56].

A total of 60 micropillars were fabricated, out of which 40 were used for quasi-static, monotonic tests and 10 for cyclic loading tests. Micropillars were compressed using an in situ indenter (Alemnis, Switzerland) [57] inside a SEM (Zeiss, Germany) operated at $5 \mathrm{kV}$, allowing precise alignment and tracking of the deformation. A diamond flat punch (Synton-MDP, Switzerland) of $10 \mu \mathrm{m}$ diameter and cone angle of $45^{\circ}$ was used to compress the micropillars in displacement control (Supplementary Fig. 5) at a rate of $5 \mathrm{~nm} / \mathrm{s}$, corresponding to a strain rate of $\sim 5 \times 10^{-4} \mathrm{~s}^{-1}$. Post-failure micrographs were taken using a HRSEM (Hitachi, Japan) operated at $3 \mathrm{kV}$ and $55^{\circ}$ specimen tilt. The displacement data were corrected for instrument frame compliance and sink-in of the micropillar using the Sneddon approach [38] (fillet radius $300 \mathrm{~nm}$ ).

Engineering stress-strain data was obtained by dividing force by the average cross sectional area and compliance-corrected displacement by the height of each micropillar and converted to true stress-strain measures, i.e. Cauchy stress $\sigma$ and logarithmic stretch $\ln U$, using the assumption of negligible volume change [58].

For the monotonic tests, samples were loaded in displacement control until failure. Yield stress was determined using the $0.2 \%$ offset rule, ultimate stress is the maximum stress in the true stress-strain curve. For cyclic loading, samples were loaded in displacement control with 5 intermittent cycles with an amplitude of $100 \mathrm{~nm}$ after every $200 \mathrm{~nm}$ of loading until failure (Supplementary Fig. 6). The apparent modulus $E_{a p p}$ was determined by fitting a line to the last of each 5 cycles. Modulus was normalized with respect to the one measured during the second cycle to minimize the influence of the toe region. Plastic strains were determined using 


$$
\ln U_{33}^{p}=\ln U_{33}-\frac{\sigma_{33}}{E_{a p p}} .
$$

For the macroscopic tests, samples were cut from the diaphyses of ovine tibiae using a diamond-coated bandsaw and subsequently lathed to a dumbbellshaped form with a diameter of $3 \mathrm{~mm}$ and an aspect ratio of 2 for the reduced section using a desktop lathe. For an overview of the tested sample geometries and the relevant bone structures, see Supplementary Fig. 7. The samples were dried for more than $48 \mathrm{~h}$ and subsequently tested in a servo-hydraulic testing device (858 Mini Bionix, MTS, USA). The machine was operated displacement controlled at a rate of $18 \mu \mathrm{m} / \mathrm{s}$ (strain rate $\approx 5 \times 10^{-4} \mathrm{~s}^{-1}$ ) with 5 intermittent cycles with an amplitude of $0.125 \%$ after every $0.25 \%$ of strain until failure. An extensometer (Epsilon Tech., USA) was attached to the reduced section with an initial gauge length of $6 \mathrm{~mm}$.

All data manipulations and statistical analysis were performed using $\mathrm{R}$ [59]. Normality of distributions was tested by visual inspection of normalized quantile-quantile (Q-Q) plots against the standard normal distribution and the Shapiro-Wilk normality test [59, 60]. Measurements are reported as mean \pm standard deviation. Significant differences were tested using twotailed t-tests. Influence of measured parameters on the data was tested by ANOVA and linear regressions using dummy coding for categorical variables. The significance threshold was chosen as $\mathrm{p}=0.01$.

\section{References}

[1] Fratzl, P. \& Weinkamer, R. Nature's hierarchical materials. Prog Mater Sci 52(8), 1263 - 1334 (2007).

[2] Weiner, S., Traub, W. \& Wagner, H. Lamellar bone: Structure-function relations. J Struct Biol 126(3), 241 - 255 (1999).

[3] Currey, J.D. Bones: Structure amd Mechanics (Princeton University Press, Princeton, 2002).

[4] Currey, J.D. The relationship between the stiffness and the mineral content of bone. J Biomech 2(4), 477 - 480 (1969).

[5] Lees, S., Tao, N.-J. \& Lindsay, S.. Studies of compact hard tissues and collagen by means of Brillouin light scattering. Connect Tissue Res 24(3-4), 187 - 205 (1990).

[6] Giraud-Guille, M.M. Twisted plywood architecture of collagen fibrils in human compact bone osteons. Calcified Tissue Int 42(3), 167-180 (1988). 
[7] Weiner, S., Arad, T., Sabanay, I. \& Traub, W. Rotated plywood structure of primary lamellar bone in the rat: Orientations of the collagen fibril arrays. Bone 20(6), 509 - 514 (1997).

[8] Martin, R.B. Porosity and specific surface of bone. Crit Rev Biomed Eng 10(3), 179 - 222 (1984).

[9] Cowin, S.C. Bone Mechanics Handbook (CRC Press, Boca Raton, 2001)

[10] Kutz, M. Standard Handbook of Biomedical Engineering $\& 3$ Design Ch. 8 (McGraw Hill, New York, 2003).

[11] Gruber, P. et al. Biomimetics - Materials, Structures and Processes. Ch. 5 (Springer, Berlin, 2011).

[12] Rho, J.-Y, Kuhn-Spearing, L. \& Zioupos, P. Mechanical properties and the hierarchical structure of bone. Med Eng Phys 20(2), 92 - 102 (1998).

[13] O'Brien, F.J., Taylor, D. \& Lee, T.C. An improved labelling technique for monitoring microcrack growth in compact bone. J Biomech 35(4), $523-526(2002)$.

[14] Sun, X., Jeon, J.H., Blendell, J. \& Akkus, O. Visualization of a phantom post-yield deformation process in cortical bone. J Biomech 43(10), 1989 - 1996 (2010).

[15] Zioupos, P., Hansen, U. \& Currey, J.D. Microcracking damage and the fracture process in relation to strain rate in human cortical bone tensile failure. J Biomech 41(14), 2932 - 2939 (2008).

[16] Gupta, H. et al. Fibrillar level fracture in bone beyond the yield point. Int J Fracture 139, 425-436 (2006).

[17] Oliver, W.C. \& Pharr, G.M. An improved technique for determining hardness and elastic modulus using load and displacement sensing indentation experiments. J Mater Res 7, 1564 - 1583 (1992).

[18] Lewis, G. \& Nyman, J.S.. The use of nanoindentation for characterizing the properties of mineralized hard tissues: State of the art review. $J$ Biomed Mater Res B 87(1), 286-301 (2008).

[19] Zysset, P.K., Guo, X.E., Hoffler, C.E., Moore, K.E. \& Goldstein, S.A. Elastic modulus and hardness of cortical and trabecular bone lamellae measured by nanoindentation in the human femur. J Biomech 32(10), 1005 - 1012 (1999). 
[20] Lucchini, R. et al. Role of damage mechanics in nanoindentation of lamellar bone at multiple sizes: Experiments and numerical modeling. J Mech Behav Biomed 4(8), 1852 - 1863 (2011).

[21] Zhang, J., Michalenko, M.M., Kuhl, E. \& Ovaert, T.C. Characterization of indentation response and stiffness reduction of bone using a continuum damage model. J Mech Behav Biomed 3(2), 189 - 202 (2010).

[22] Chen, X., Ogasawara, N., Zhao, M. \& Chiba, N. On the uniqueness of measuring elastoplastic properties from indentation: The indistinguishable mystical materials. J Mech Phys Solids 55(8), 1618 - 1660 (2007).

[23] Howie, P.R., Korte, S. \& Clegg, W.J. Fracture modes in micropillar compression of brittle crystals. J Mater Res, 27(1), 141 - 151 (2012).

[24] Michler, J., Wasmer, K., Meier, S., Ostlund, F. \& Leifer, K. Plastic deformation of gallium arsenide micropillars under uniaxial compression at room temperature. Appl Phys Lett 90(4), 043123 - 043123-3 (2007).

[25] Bažant, Z. Scaling theory for quasibrittle structural failure. P Natl A Sci USA 101(37), 13400 - 13407 (2004).

[26] Östlund, F. et al. Brittle-to-ductile transition in uniaxial compression of silicon pillars at room temperature. Adv Funct Mater 19(15), 2439 - 2444 (2009).

[27] Östlund, F. et al. Ductile-brittle transition in micropillar compression of GaAs at room temperature. Philos Mag 91(7-9), 1190 - 1199 (2011).

[28] Griffith, A.A. The phenomena of flow and rupture in solids. Philos T $R$ Soc A 221, 163-198 (1921).

[29] Hengsberger, S., Kulik, A. \& Zysset, P.K. Nanoindentation discriminates the elastic properties of individual human bone lamellae under dry and physiological conditions. Bone, 30(1), 178 - 184 (2002).

[30] Carnelli, D., Lucchini, R., Ponzoni, M., Contro, R. \& Vena, P. Nanoindentation testing and finite element simulations of cortical bone allowing for anisotropic elastic and inelastic mechanical response. $J$ Biomech, 44(10), 1852 - 1858 (2011).

[31] Reisinger, A.G., D.H. Pahr, D.H. \& Zysset, P.K. Principal stiffness orientation and degree of anisotropy of human osteons based on nanoindentation in three distinct planes. J Mech Behav Biomed 4(8), 2113 2127 (2011). 
[32] Franzoso, G. \& Zysset, P.K. Elastic anisotropy of human cortical bone secondary osteons measured by nanoindentation. J Biomech Eng - T ASME 131(11), 117001 (2009).

[33] Chamay, A. Mechanical and morphological aspects of experimental overload and fatigue in bone. J Biomech 3(3), 263-270 (1970).

[34] Krajcinovic, D., Trafimow, J. \& Sumarac, D. Simple constitutive model for a cortical bone. J Biomech 20(8), 779-784 (1987).

[35] Pearce, A.I., Richards, R.G., Milz, S., Schneider, E. \& Pearce, S.G. Animal models for implant biomaterial research in bone: a review. Eur Cells Mater 13, 1 - 10 (2007).

[36] Ravaglioli, A. et al. Mineral evolution of bone. Biomaterials 17(6), $617-622(1996)$.

[37] Reilly, D.T. \& Burstein, A.H. The elastic and ultimate properties of compact bone tissue. J Biomech 8(6), 393 - 405 (1975).

[38] Zhang, H., Schuster, B.E., Wei, Q. \& Ramesh, K.T. The design of accurate micro-compression experiments. Scripta Mater 54(2), 181 186 (2006).

[39] Fantner, G.E. et al. Hierarchical interconnections in the nano-composite material bone: Fibrillar cross-links resist fracture on several length scales. Compos Sci Technol 66(9), 1205-1211 (2006).

[40] Koester, K.J., Ager, J.W. \& Ritchie, R.O. The true toughness of human cortical bone measured with realistically short cracks. Nat Mater $\mathbf{7}(\mathbf{8})$, 672-677 (2008).

[41] Peterlik, H., Roschger, P., Klaushofer, K. \& Fratzl, P. From brittle to ductile fracture of bone. Nat Mater 5(1), 52 - 55 (2006).

[42] Poundarik, A.A. et al. Dilatational band formation in bone. P Natl A Sci USA 109(47), 19178 - 19183 (2012).

[43] Tai, K. Ulm, F.-J. \& Ortiz, C. Nanogranular origins of the strength of bone. Nano Lett 6(11), 2520 - 2525 (2006).

[44] Mercer, C., He, M.Y., Wang, R. \& Evans, A.G. Mechanisms governing the inelastic deformation of cortical bone and application to trabecular bone. Acta Biomater 2(1), 59 - 68 (2006).

[45] Gupta, H. et al. Intrafibrillar plasticity through mineral/collagen sliding is the dominant mechanism for the extreme toughness of antler bone. J Mech Behav Biomed 28, 366 - 382 (2013). 
[46] Hayes, W.C. \& Carter, D.R. Postyield behavior of subchondral trabecular bone. J Biomed Mater Res 10, 537-544 (1976).

[47] Schaffler, M.B., Choi, K. \& Milgrom, C. Aging and matrix microdamage accumulation in human compact bone. Bone 17(6), $521-525$ (1995).

[48] Carter, D.R. \& Hayes, W.C. Compact bone fatigue damage: A microscopic examination. Clin Orthop Relat R 127, 265-274 (1977).

[49] Martin, R.B. \& Burr, D.B. Structure, Function, and Adaptation of Compact Bone (Raven Press, New York, 1989).

[50] Currey, J.D. Stress concentrations in bone. Q J Microsc Sci 103(1), 111-133 (1962).

[51] Zysset, P.K. A Constitutive Law for Trabecular Bone. (PhD thesis, Ecole Polytechnique Federale de Lausanne, Lausanne, 1994).

[52] Fischer-Cripps, A.C. Nanoindentation (Springer, New York, 2002).

[53] Nyman, J.S. et al. The influence of water removal on the strength and toughness of cortical bone. J Biomech 39(5), 931 - 938 (2006).

[54] Wolfram, U., Wilke, H.-J. \& Zysset, P.K. Rehydration of vertebral trabecular bone: Influences on its anisotropy, its stiffness and the indentation work with a view to age, gender and vertebral level. Bone 46(2), 348 - 354 (2010).

[55] Ziegler, J.F. \& Biersack, J.P. The Stopping and Range of Ions in Matter (Springer, New York, 1985).

[56] Nalla, R. et al. Ultrastructural examination of dentin using focused ionbeam cross-sectioning and transmission electron microscopy. Micron 36, $672-680$ (2005).

[57] Rabe, R. et al. Observation of fracture and plastic deformation during indentation and scratching inside the scanning electron microscope. Thin Solid Films 469, 206 - 213 (2004).

[58] Ashby, M. \& Jones, D. Engineering Materials (Pergamon Press, Oxford, 1980).

[59] R Development Core Team. R: A Language and Environment for Statistical Computing. (R Foundation for Statistical Computing, Vienna, 2008).

[60] Shapiro, S.S. \& Wilk, M.B. An analysis of variance test for normality (complete samples). Biometrika, 52(3/4), 591 - 611 (1965). 


\section{Acknowledgments}

The authors would like to thank Mohammad Mirzaali for his help with the specimen preparation, Ivo Utke, $\mathrm{PhD}$, for the discussions about ionmatter interactions and SRIM, Caspar Schwiedrzik, $\mathrm{PhD}$ for his valuable comments on the manuscript, and Damian Frey and Gerhard Buerki for technical assistance with the in situ indenter and FIB milling.

\section{Author contributions}

The initial planning of the study was done by JS, RR, JM and PZ. FIB was operated by RR. Micropillar compressions and SEM imaging were performed by JS and RR, Raman measurements and interpretation by VL, Monte Carlo simulations and microindentations by JS, macroscopic tests by JS, UW and AB. Data analysis was performed by JS and RR with assistance of UW, interpretation in cooperation with JM and PZ. Modeling was performed by JS and PZ. The manuscript was written by JS with contributions of RR, $\mathrm{AB}, \mathrm{VL}, \mathrm{UW}, \mathrm{JM}$ and PZ. 
Figure 1: Experimental curves and postyield behaviour of monotonic micropillar compression tests. True stress-strain curves (top) and postyield behavior normalized with ultimate stress as a function of plastic strain (bottom) of monotonic micropillar compression in a) axial and b) transverse direction. Observed yield points are indicated by hollow circles and ultimate points by full circles. 
Figure 2: Observed failure modes of bone on the micro- and macroscale. HRSEM micrographs taken after testing, FIB cross-sections and photographs showing failure modes encountered in compression tests a) of micropillars in axial and b) transverse directions as well as c) of macroscopic specimens. Micropillars mostly deformed homogeneously and failed by development of slip planes (a1, b1-b3). A minority of the axial pillars failed by mushrooming (a2) or axial splitting (a3), which is a brittle failure mode. Scale bars for a1-a3 and b1-b3 represent $2 \mu \mathrm{m}$, for c1-c4 $4 \mathrm{~mm}$. 
Figure 3: Rheological model describing the mechanical response of bone under compression. A parallel array of elastic springs in series with plastic pads failing at a ultimate plastic strain (Supplementary Fig. 8 and 9 ). On the microscale, heterogeneity is governed by Gaussian distributions of the material properties modulus, yield stress, hardening modulus and ultimate plastic strain (Supplementary Tab. 1) identified from the micropillar compression tests. For the macroscale, the presence of cracks, defects and interfaces increases tissue heterogeneity leading to a reduction of strength and ductility. Therefore, uniform distributions between 0 and the microscopic properties are used (Supplementary Fig. 10) to describe variability in strength and ultimate plastic strain corresponding to the pre-existence of defects and microcracks with different lengths from 0 to a critical length. Further details are given in the supplementary material. 
Figure 4: Model predictions, experimental curves and normalized apparent modulus evolution of cyclic compression tests on the micro- and macroscale. a) Model predictions, experimental true stressstrain curves in b) axial and c) transverse direction and d) normalized apparent modulus evolution as a function of plastic strain of cyclic micropillar (top) and macroscopic (bottom) compression. 
Figure 5: Dominant failure mechanism observed on the microscale. Overlay of SEM micrographs of micropillars in axial (top, left) and in transverse (top, right) direction failed by shearing with HR images of the slip planes and schematical drawings (bottom) depicting the distinct failure mechanisms depending on the fibrillar orientation. Scale bars on the top represent $2 \mu \mathrm{m}$, on the bottom $0.5 \mu \mathrm{m}$. 
Table 1: Mean \pm standard deviation of the indentation modulus $E^{*}$, indentation hardness $H_{I T}$, elastic $W_{e l}$ and total Work $W_{t o t}$ as well as number of experiments $N$ for wet and dry indentations in axial and transverse directions.

\begin{tabular}{lcccccc} 
Hydr. & Direct. & $E^{*} / \mathrm{GPa}$ & $H_{I T} / \mathrm{GPa}$ & $W_{\text {el }} / \mathrm{pJ}$ & $W_{\text {tot }} / \mathrm{pJ}$ & $N$ \\
\hline Dry & Axial & $27.5 \pm 2.2$ & $1.01 \pm 0.13$ & $1837 \pm 258$ & $8069 \pm 1012$ & 50 \\
Dry & Transv. & $19.0 \pm 1.8$ & $0.67 \pm 0.08$ & $1636 \pm 214$ & $6482 \pm 745$ & 72 \\
Wet & Axial & $22.8 \pm 1.6$ & $0.60 \pm 0.11$ & $1083 \pm 249$ & $4849 \pm 907$ & 67 \\
Wet & Transv. & $14.5 \pm 1.6$ & $0.51 \pm 0.08$ & $1313 \pm 204$ & $4125 \pm 756$ & 83 \\
\hline
\end{tabular}




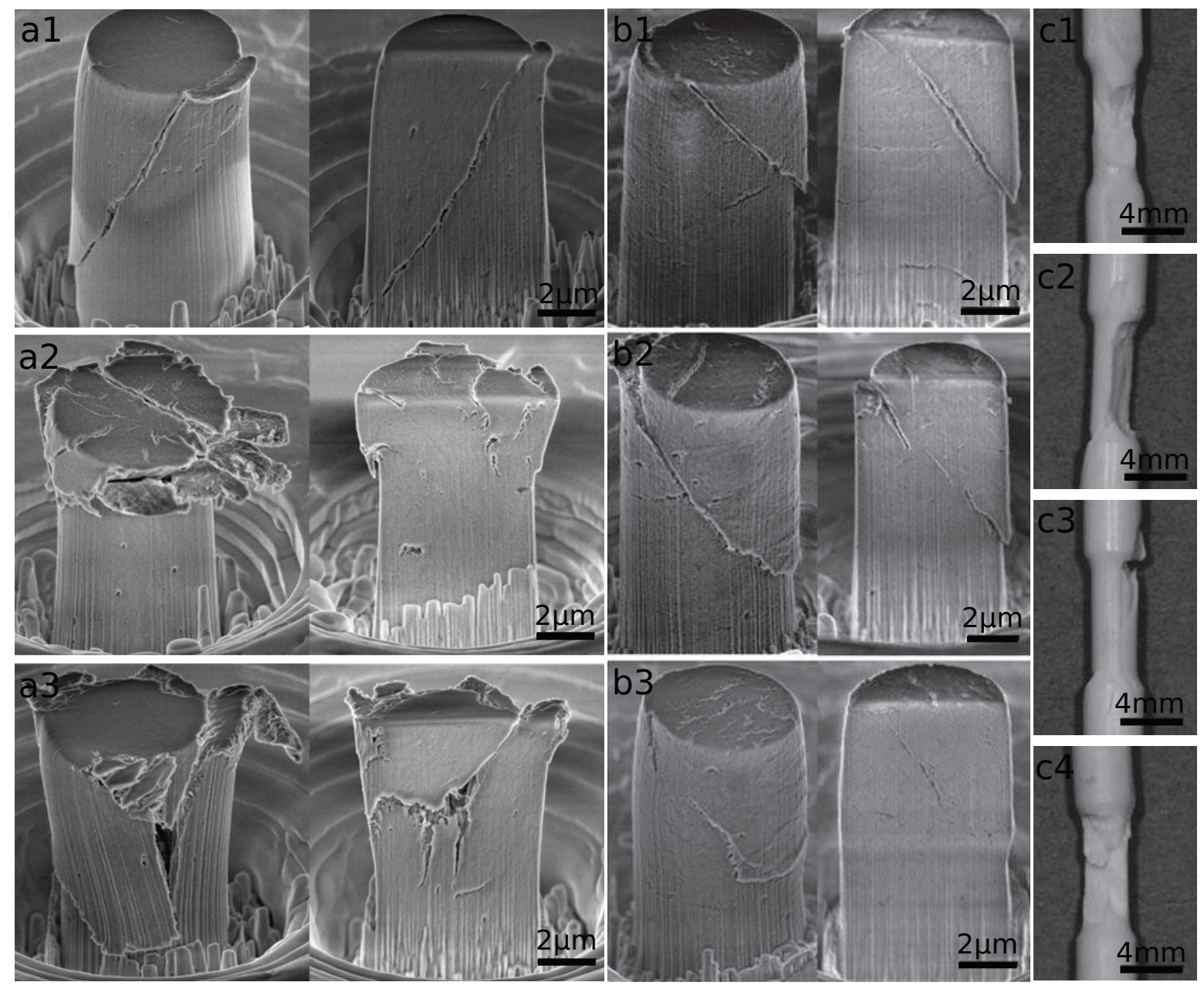



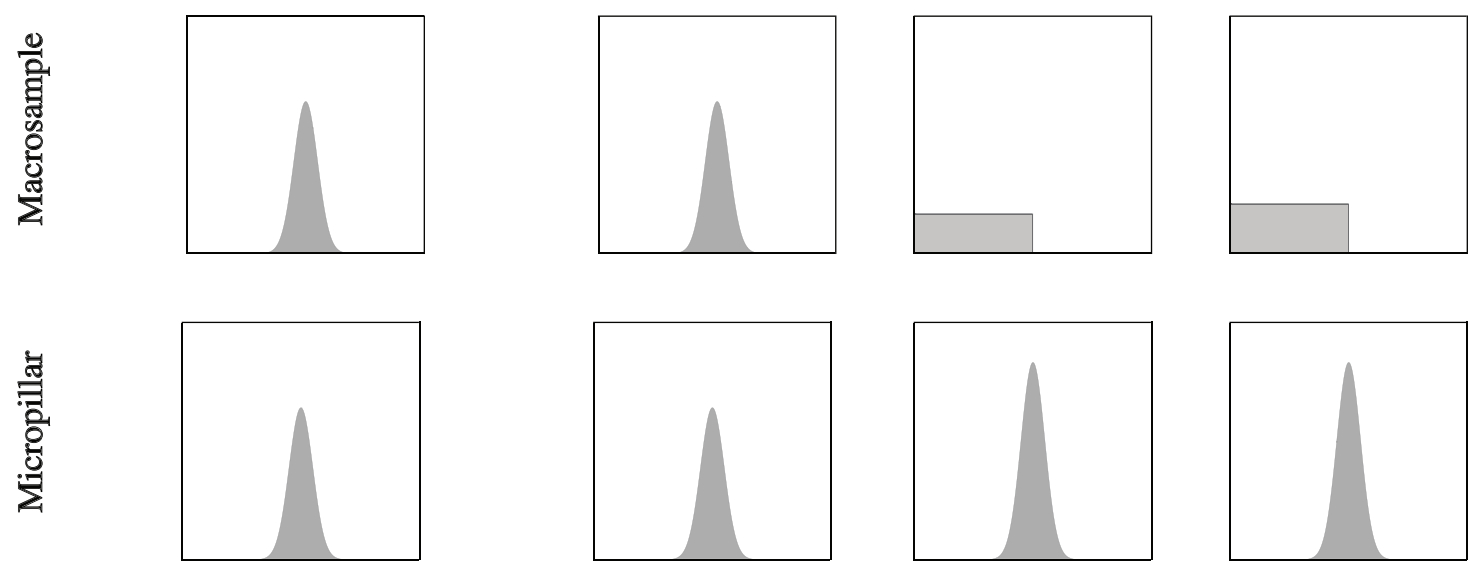

$\mathrm{E}_{\mathrm{n}}$

$\mathrm{H}_{\mathrm{n}}$

$\ln U_{n}^{y}$

$\ln U_{\mathrm{n}}^{\mathrm{p}, \mathrm{ult}}$

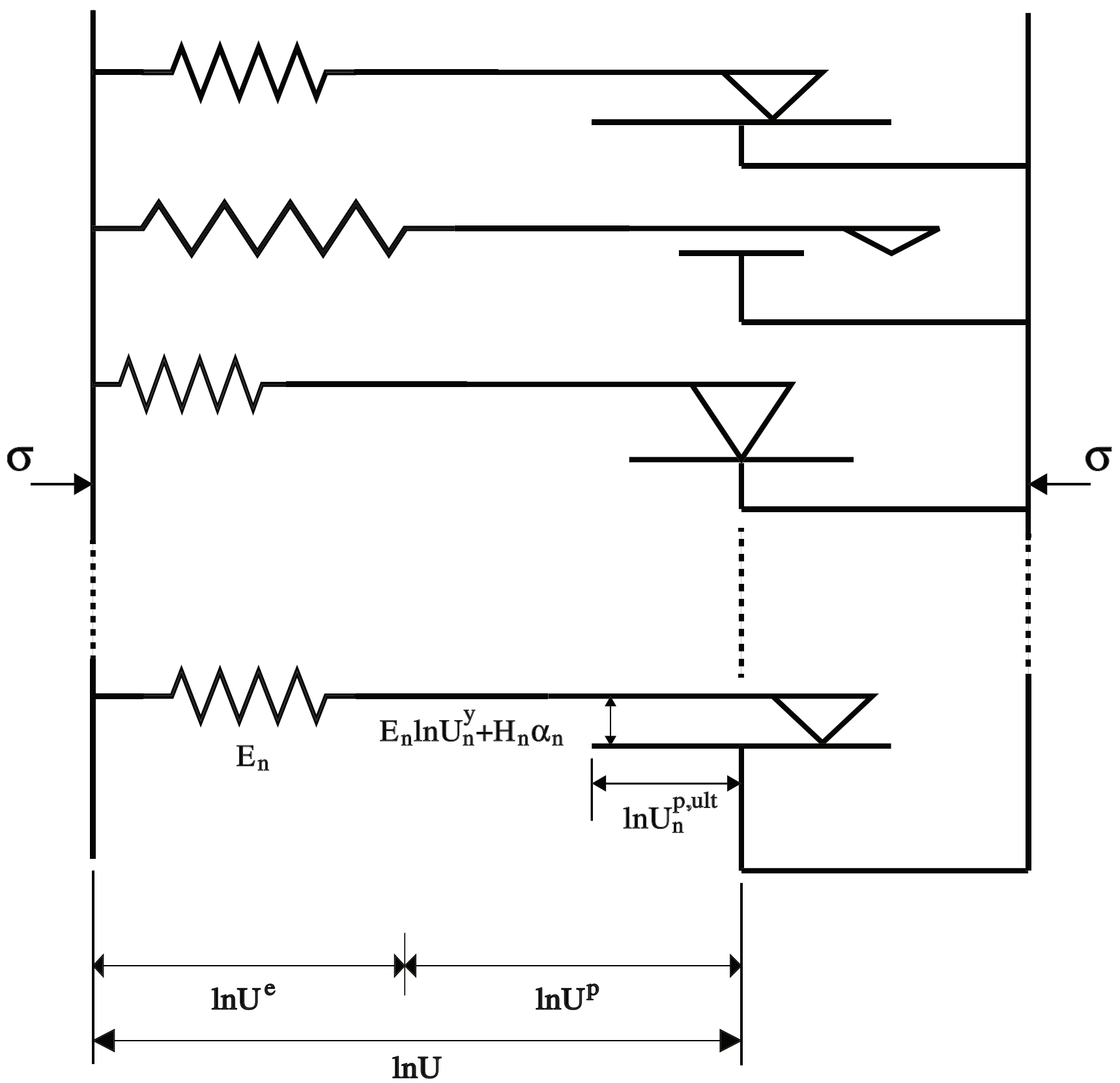




\title{
Supplementary Information to 'In situ micropillar compression of lamellar bone reveals superior strength and ductility but no damage'
}

\author{
Jakob Schwiedrzik, Rejin Raghavan, Alexander Bürki, Victor LeNader, Uwe \\ Wolfram, Johann Michler, and Philippe Zysset
}

\section{Sample preparation, chemical analysis, testing setup and data analysis}

Sheep tibiae were acquired from a local butcher shop. Ovine bone is mostly primary, features less porosity and a more homogeneous but similar mineralization to human bone $(1 ; 2)$, making it an attractive model for human bone on the microlevel. Axial and transverse specimens were cut from the diaphyses with a diamond-coated band saw (Exact, Germany). They were embedded in, but not infiltrated by, polymethylmethacrylate (PMMA) and subsequently air-dried (3) for more than 48 h. Finally, they were ultramilled (Polycut E, Reichert-Jung, Germany) to obtain a flat surface for the Raman, microindentation and micropillar compression experiments.

Raman spectroscopy was performed on a transverse control sample in dry condition using an upright Raman microscope (Nova Spectra, ND-MDT, Russia) featuring a laser source with a wavelength of $633 \mathrm{~nm}, 5 \mathrm{~mW}$ power and a 100x objective with a numerical aperture of 0.95. Spectra were recorded at a spectral resolution of $3 \mathrm{~cm}^{-1}$. The exposure time was $1 \mathrm{~s}$.

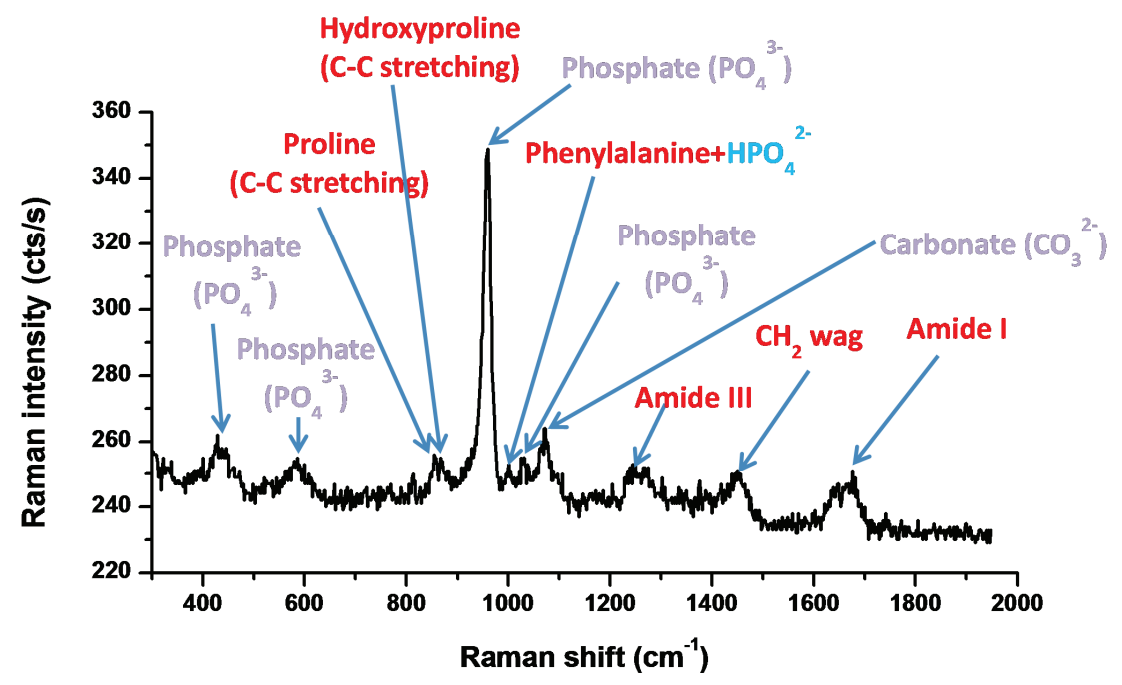

Supplementary Figure 1: Raman spectrum of ovine cortical bone. Representative spectrum from an osteonal region with identified peaks. 
The Raman spectra corresponded well to the literature (4) and all characteristic peaks could be identified. Based on previous work on bone quality assessment using vibrational spectroscopy $(5 ; 6)$, three physicochemical properties of the tissue were investigated: mineralization, i.e. the ratio of mineral to organic components, substitution of carbonate ions in phosphate positions, and mineral cristallinity, i.e. the orderliness of the crystal lattice. The calculation of these properties is explained in Fig. 2. Mineralization was found to be $11.0 \pm 1.1$, carbonate substitution $7.6 \pm 0.8$, and crystallinity $19.6 \pm 0.4 \mathrm{~cm}^{-1}(\mathrm{~N}=10)$. This is reasonably similar to the results of Yerramshetty et al. (6), who found a mineralization of 8.0 to 10.0, carbonation of 4.5 to 5.3 and crystallinity of 16.7 to $19.2 \mathrm{~cm}^{-1}$ in hydrated human femoral bone. It can therefore be concluded that the chemical composition of the tested samples was in the expected range and that ovine bone is indeed a reasonable model for human bone on the microscale.

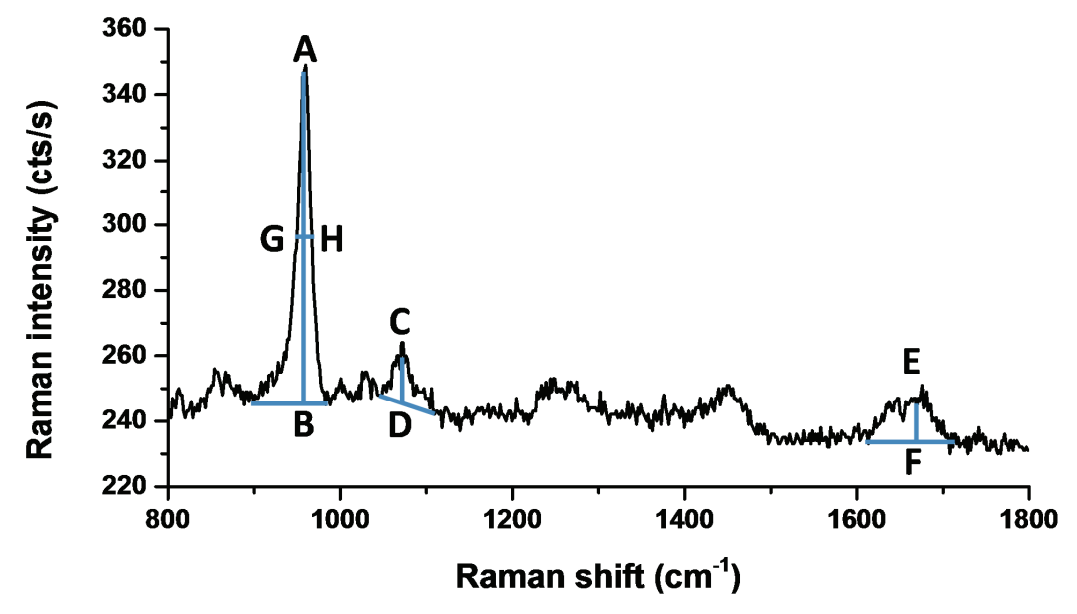

Supplementary Figure 2: Measurements on Raman spectrum of ovine cortical bone in order to measure mineralization, carbonate substitution and crystallinity based on (5). Mineralization=AB/EF, Substitution $=\mathrm{AB} / \mathrm{CD}$, Crystallinity $=\mathrm{GH}$.

Microindentations were performed in dry and wet state in osteonal regions with an Ultra Nano Hardness Tester (UNHT, CSM Instruments, Switzerland). For the wet nanoindentations, control samples were rehydrated for at least $1.5 \mathrm{~h}$ in Hank's balanced saline solution (HBSS) following the protocol of Wolfram et al. (3) and subsequently indented in a HBSS submersion. A trapezoidal protocol in load control up to a maximum depth of $1 \mu \mathrm{m}$ with a loading rate of $100 \mathrm{mN} / \mathrm{min}$, holding time of $30 \mathrm{~s}$ (3) and unloading rate of $400 \mathrm{mN} / \mathrm{min}$ was chosen to minimize the effects of creep. Indentation modulus and hardness, elastic and total work were extracted using standard methods $(7 ; 8 ; 3)$. Indentation modulus is recovered from the reduced modulus $E_{r}(7)$ by the equation

$$
E^{*}=\left(\frac{1}{E_{r}}-\frac{1-\nu_{i}^{2}}{E_{i}}\right)^{-1}
$$

for known isotropic constants $E_{i}$ and $\nu_{i}$ of the indenter tip. Indentation hardness is defined as the maximum load divided by the contact area at maximum depth, elastic and total work as the area under the unloading curve and the loading and holding curves. A transversely isotropic stiffness 
tensor was fitted to the indentation moduli using the method of Franzoso et al. (9) to obtain estimates for apparent moduli. For the wet indentations in transverse direction, a swelling effect similar to the one reported in (10) was found that increases surface roughness and affects the experimental curves. This data was therefore not used for further analysis.

After indentation, the samples were extracted from the embedding material and glued on SEM stubs using silver adhesive. A $50 \mathrm{~nm}$ thick $\mathrm{Au}-\mathrm{Pd}$ film was sputtered on the specimens to minimize drift due to charging. A dual beam FIB workstation (Tescan Lyra, Czech Republic) operated at $30 \mathrm{kV}$ was used to machine micropillars of $5 \mu \mathrm{m}$ diameter and aspect ratio of 2 in osteonal regions, and to section them after testing. Micropillars were aligned with the longitudinal and circumferential directions of the respective osteons. The micropillars were machined to the desired dimensions in three major steps: First, circular trenches of $50 \mu \mathrm{m}$ diameter were machined with $6000 \mathrm{pA}$ to obtain $10 \mu \mathrm{m}$ diameter posts, which were milled down to $6 \mu \mathrm{m}$ with $1000 \mathrm{pA}$ and polished to $5 \mu \mathrm{m}$ with $300 \mathrm{pA}$.
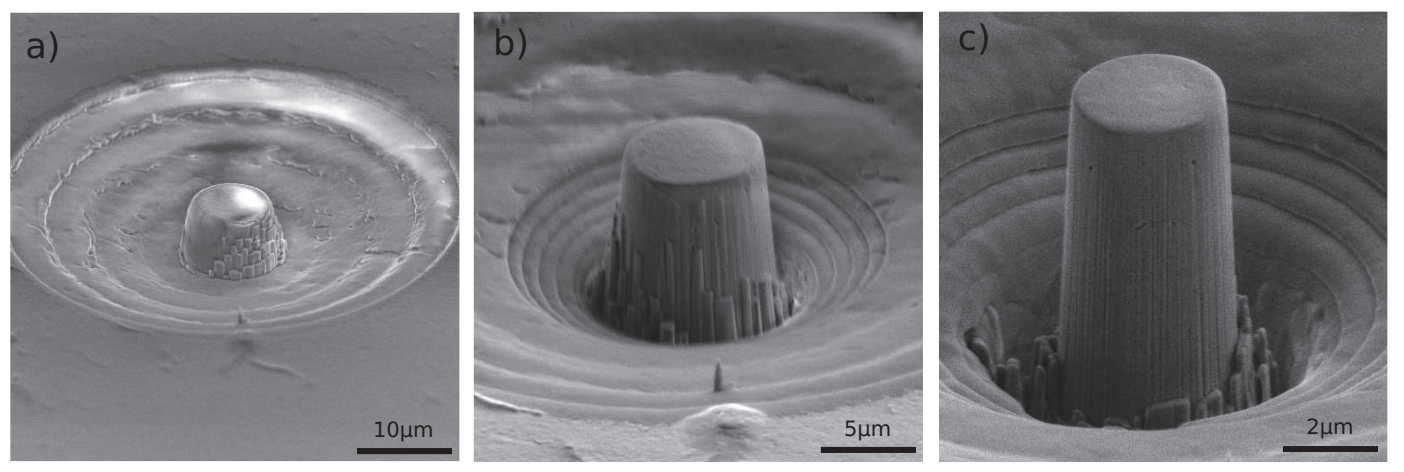

Supplementary Figure 3: SEM micrographs showing the micropillar machining process. a) Micropillar after trench milling with 6000 pA, b) after intermediate step with 1000 pA, c) after final polishing step with $300 \mathrm{pA}$.

FIB induced damage and Gallium implantation at the surface are common problems in micropillar compression studies. In order to assess these effects, Monte Carlo simulations using the software SRIM (11) with an incidence angle of $3^{\circ}, 30 \mathrm{keV} \mathrm{Ga}^{+}$ions, and the material composition for cortical bone ICRU-119 from the software's compound library were performed. They showed that damage on the side of the pillars are confined to a layer of $25 \mathrm{~nm}$ and can therefore be neglected (12) from a mechanical point of view. The calculated ion range is $8.7 \pm 5.9 \mathrm{~nm}$ and therefore also neglectable. These results are in line with the study of Nalla et al. (13), who report no significant FIB damage for mineralized tissues.

A total of 60 micropillars were fabricated, out of which 40 were used for quasi-static, monotonic tests and 10 for cyclic loading tests. Micropillars were compressed using an in situ indenter (Alemnis, Switzerland) (14) inside a SEM (Zeiss, Germany) operated at $5 \mathrm{kV}$, allowing precise alignment and tracking of the deformation. A diamond flat punch (Synton-MDP, Switzerland) of $10 \mu \mathrm{m}$ diameter and cone angle of $45^{\circ}$ was used to compress the micropillars in displacement control at a rate of $5 \mathrm{~nm} / \mathrm{s}$, corresponding to a strain rate of $\sim 5 \times 10^{-4} \mathrm{~s}^{-1}$. A schematic drawing of the experimental setup is shown below.

Post-failure micrographs were taken using a HRSEM (Hitachi, Japan) operated at $3 \mathrm{kV}$ and $55^{\circ}$ 


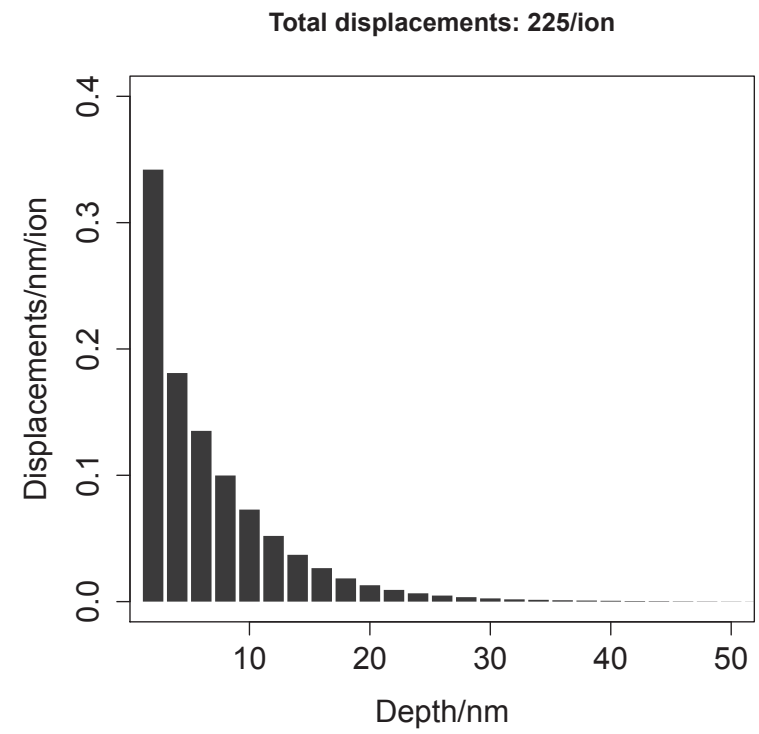

Supplementary Figure 4: FIB damage distribution determined by means of a Monte Carlo simulation using the software SRIM. Atomar displacements/nm/ion due to ion bombardment and damage cascade as a function of radial distance from the surface $/ \mathrm{nm}$.

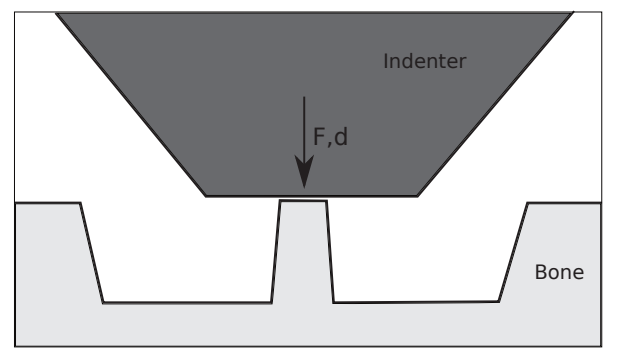

Supplementary Figure 5: Sketch of the experimental setup of the micropillar compression experiments. A flat punch indenter is placed on top of the micropillar that is surrounded by a trench. The indenter is then moved downward and tip displacement as well as the axial force are recorded.

specimen tilt. The displacement data was corrected for instrument frame compliance and sink-in of the micropillar using the so called Sneddon approach proposed by Zhang et al. (15) with a fillet radius of $300 \mathrm{~nm}$.

Engineering stress-strain data was obtained by dividing force by the average cross sectional area and compliance-corrected displacement by the height of each micropillar and converted to true stress-strain measures, i.e. Cauchy stress $\sigma$ and logarithmic stretch $\ln U$, using the assumption of negligible volume change (16).

$$
\begin{aligned}
& \sigma=\sigma_{E n g}\left(1+\epsilon_{E n g}\right) \\
& \ln U=\ln \left(1+\epsilon_{E n g}\right)
\end{aligned}
$$

For the monotonic tests, samples were loaded in displacement control until failure. Yield stress 
was determined using the $0.2 \%$ offset rule, ultimate stress is the maximum stress in the true stressstrain curve. For cyclic loading, samples were loaded in displacement control with 5 intermittent cycles with an amplitude of $100 \mathrm{~nm}$ after every $200 \mathrm{~nm}$ of loading until failure. The apparent modulus $E_{a p p}$ was determined by fitting a line to the last of each 5 cycles. Modulus was normalized with respect to the one measured during the second cycle to minimize the influence of the toe region. A schematic drawing explaining the cyclic micropillar compression analysis is shown below.

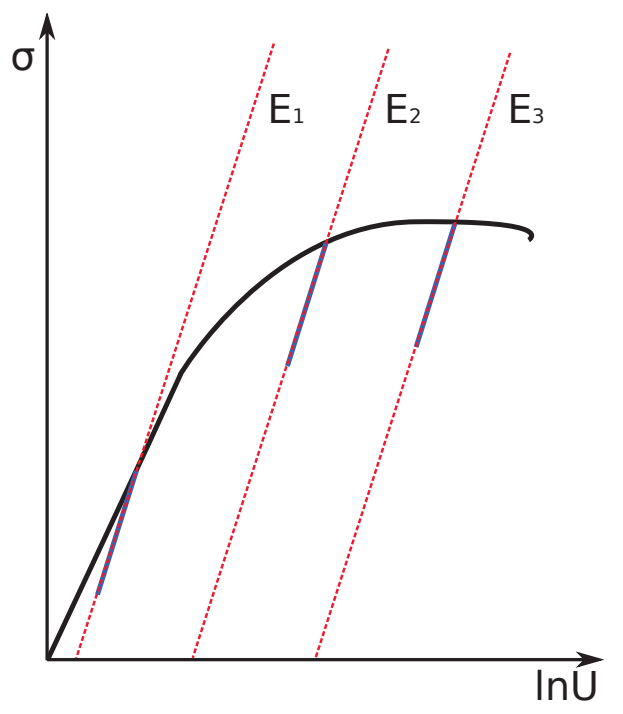

Supplementary Figure 6: Sketch depicting the analysis of cyclic experiments. Monotonic loading phases are drawn in black, intermittent cycles in blue. Apparent moduli $E_{i}$ were measured during every cycle and divided by the apparent modulus of the intermittent cycle in the elastic phase $E_{1}$.

Plastic strains were determined using

$$
\ln U_{33}^{p}=\ln U_{33}-\frac{\sigma_{33}}{E_{a p p}} .
$$

For the macroscopic tests, samples were cut from the diaphyses of ovine tibiae using a diamondcoated bandsaw and subsequently lathed to a dumbbell-shaped form with a diameter of $3 \mathrm{~mm}$ and an aspect ratio of 2 for the reduced section using a desktop lathe. The samples were dried for more than $48 \mathrm{~h}$ and subsequently tested in a servo-hydraulic testing device (858 Mini Bionix, MTS, USA). The machine was operated displacement controlled at a rate of $18 \mu \mathrm{m} / \mathrm{s}$ (strain rate $\approx 5 \times 10^{-4} \mathrm{~s}^{-1}$ ) with 5 intermittent cycles with an amplitude of $0.125 \%$ after every $0.25 \%$ of strain until failure. An extensometer (Epsilon Tech., USA) was attached to the reduced section with an initial gauge length of $6 \mathrm{~mm}$. True stress-strain data and moduli were obtained the same way as described for the cyclic micropillar experiments.

All data manipulations and statistical analysis were performed using R (17). Normality of distributions was tested by visual inspection of normalized quantile-quantile plots against the standard normal distribution and the Shapiro-Wilk normality test $(17 ; 18)$. Measurements are reported as mean \pm standard deviation. Significant differences were tested using two-tailed t-tests. Influence of measured parameters on the data was tested by ANOVA and linear regressions using dummy coding for categorical variables. The significance threshold was chosen as $p=0.01$. 


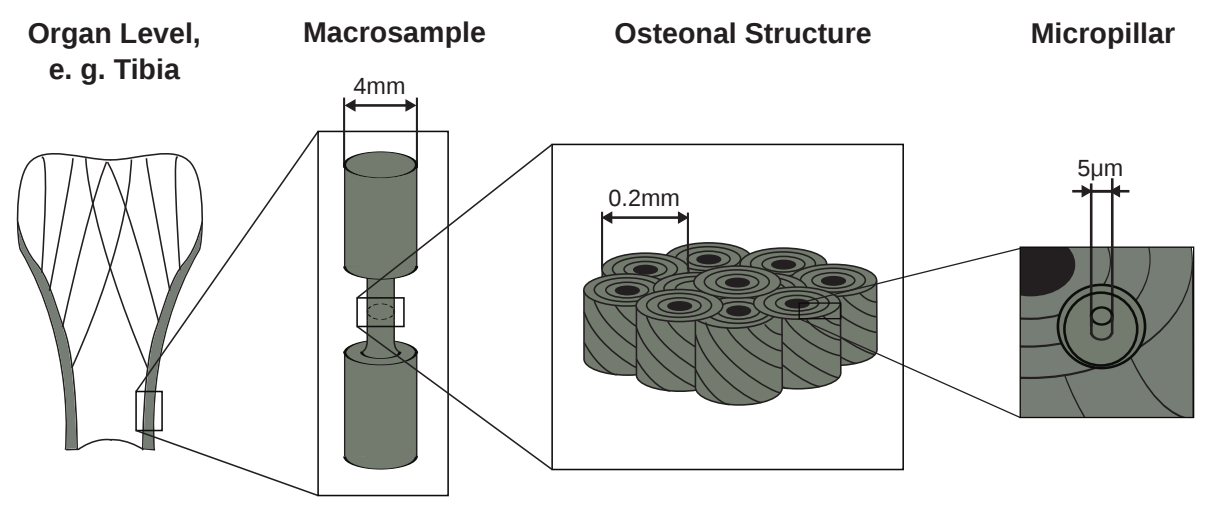

Supplementary Figure 7: Tested samples and relevant bone structures. Dumb-bell shaped macrosamples extracted from the diaphysis of ovine tibiae and micropillars on the length scale of osteonal lamellae.

\section{Rheological model}

The rheological model consists of an elastic spring in series with a plastic slider that breaks beyond a given ultimate plastic strain. The series arrangement leads to an additive decomposition of the elastic and plastic strains

$$
\ln U=\ln U^{e}+\ln U^{p}
$$

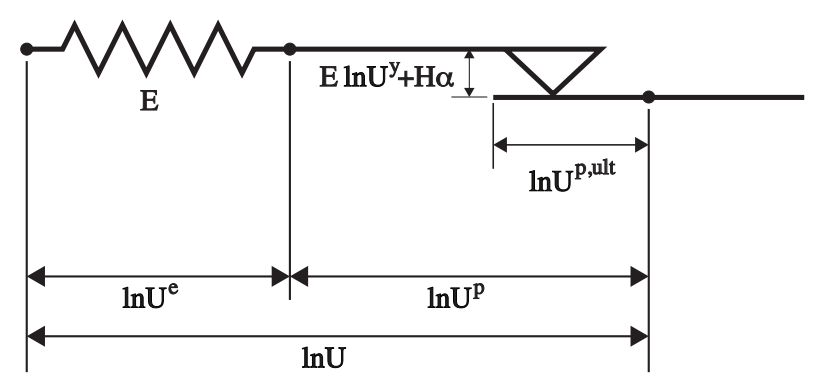

Supplementary Figure 8: Underlying rheological model and constitutive law: Elasto-plastic material with linear hardening and brittle failure when the slider quits the support after passing $\ln U^{p, u l t}$.

The spring represents a proportional relationship between the stress and the elastic strain and leads to the free energy:

$$
\begin{aligned}
\psi\left(\ln U, \ln U^{p}\right)= & \left(1-\mathcal{H}\left(-\ln U^{p}-\ln U^{p, u l t}\right)\right) \frac{1}{2} E\left(\ln U-\ln U^{p}\right)^{2} \\
& -\mathcal{H}\left(\ln U^{p}-\ln U^{p, u l t}\right) \frac{1}{2} E\left(\ln U-\ln U^{p}\right)^{2}
\end{aligned}
$$

where $E$ is Young's modulus, $\ln U^{p, u l t}$ is the ultimate or failure strain of the plastic slider and $\mathcal{H}$ is the Heaviside unit step function.

Remark 1. This formulation of the free energy corresponds to a discontinuous damage model with

$$
D=\mathcal{H}\left(-\ln U^{p}-\ln U^{p, u l t}\right)+\mathcal{H}\left(\ln U^{p}-\ln U^{p, u l t}\right)
$$


The total stress is given by the derivative of the free energy with respect to the total strain:

$$
\begin{aligned}
\sigma\left(\ln U, \ln U^{p}\right)= & \frac{\partial \psi}{\partial \ln U} \\
= & \left(1-\mathcal{H}\left(-\ln U^{p}-\ln U^{p, u l t}\right)\right) E\left(\ln U-\ln U^{p}\right) \\
& -\mathcal{H}\left(\ln U^{p}-\ln U^{p, u l t}\right) E\left(\ln U-\ln U^{p}\right)
\end{aligned}
$$

The plastic stress is the derivative of the free energy with respect to plastic strain:

$$
\begin{aligned}
\sigma^{p}\left(\ln U, \ln U^{p}\right)= & -\frac{\partial \psi}{\partial \ln U^{p}} \\
= & \delta\left(-\ln U^{p}-\ln U^{p, u l t}\right) \frac{1}{2} E\left(\ln U-\ln U^{p}\right)^{2} \\
& +\delta\left(\ln U^{p}-\ln U^{p, u l t}\right) \frac{1}{2} E\left(\ln U-\ln U^{p}\right)^{2}+\sigma
\end{aligned}
$$

where $\delta$ is the Dirac delta function.

The behavior of the plastic slider is described by a yield function for the plastic stress,

$$
f\left(\sigma^{p}\right)=\left|\sigma^{p}\right|-E \ln U^{y}-H \alpha
$$

with the classical Kuhn-Tucker conditions for $f$ and $\dot{\lambda}=\left|\ln \dot{U}^{p}\right|$

$$
f \leq 0 \quad \dot{\lambda} \geq 0 \quad f \dot{\lambda}=0
$$

where $\ln U^{y}$ is the yield strain, $H$ is the hardening modulus and the accumulated plastic strain $\alpha$ is expressed by:

$$
\alpha=\int_{0}^{t}\left|\ln \dot{U}^{p}\right| d \tau
$$

The plastic stress is finally given by

$$
\sigma^{p}= \begin{cases}-E \ln U^{y}-H \alpha & \text { if } \ln \dot{U}^{p}<0 \\ {\left[-E \ln U^{y}-H \alpha ; E \ln U^{y}+H \alpha\right]} & \text { if } \ln \dot{U}^{p}=0 \\ E \ln U^{y}+H \alpha & \text { if } \ln \dot{U}^{p}>0\end{cases}
$$

From an algorithmic point of view, computation of the plastic strain flow for a new total strain $\ln U_{n+1}$ can be initiated with a trial stress based on the plastic strain of the previous step $n$ :

$$
\sigma_{t}^{p}=E\left(\ln U_{n+1}-\ln U_{n}^{p}\right)
$$

If the yield criterion is respected for that plastic state

$$
f\left(\sigma_{t}^{p}\right) \leq 0
$$

then the total stress reduces to

$$
\sigma_{n+1}=\sigma_{t}^{p}
$$


If the yield criterion is violated

$$
f\left(\sigma_{t}^{p}\right)>0
$$

then the implicit projection of the plastic stress on the yield criterion provides an equation for $\ln U_{n+1}^{p}$

$$
f\left(\sigma^{p}\left(\ln U_{n+1}, \ln U_{n+1}^{p}\right)\right)=0
$$

The solution of the above equation is:

$$
\ln U_{n+1}^{p}=\ln U_{n}^{p}+\operatorname{sign}\left(\sigma_{t}^{p}\right) \frac{\left|\sigma_{t}^{p}\right|-\left(E \ln U^{y}+H \alpha_{n}\right)}{E+H}
$$

and the total stress is updated with

$$
\sigma_{n+1}=E\left(\ln U_{n+1}-\ln U_{n+1}^{p}\right)
$$

In case of failure of the plastic slider, i.e. $\left|\ln U_{n+1}^{p}\right| \geq \ln U^{p, u l t}$, the total stress vanishes abruptly

$$
\sigma_{n+1}=0
$$

The dissipation of the model is the usual dissipation of the plastic slider plus the elastic energy of the spring released by breakage of the slider when $\left|\ln U^{p}\right|=\ln U^{p, u l t}$,

$$
\begin{aligned}
\phi\left(\ln \dot{U}^{p}\right)= & \sigma^{p} \ln \dot{U}^{p} \\
= & \sigma \ln \dot{U}^{p}+\delta\left(\ln U^{p}+\ln U^{p, u l t}\right) \frac{1}{2} E\left(\ln U-\ln U^{p}\right)^{2} \\
& +\delta\left(\ln U^{p}-\ln U^{p, u l t}\right) \frac{1}{2} E\left(\ln U-\ln U^{p}\right)^{2}
\end{aligned}
$$

and vanishes beyond failure of the plastic slider.

Remark 2. The model degenerates into an elastic material for $\ln U^{y} \rightarrow \infty$, a perfectly elastoplastic material for $\ln U^{p, u l t} \rightarrow \infty$ or into an elastic and perfectly brittle material for $\ln U^{p, u l t}=0$. In the case of $\ln U^{p, u l t}=0$, the damage model of Krajcinovic (19) is obtained for a homogeneous distribution governing the yield stress.

The model consists of a parallel array of 100 parallel elements. The total response is given by the summation over all elements. For the micropillars, all material properties are governed by the experimentally measured normal distributions. On the other hand, a macroscopic bone sample will contain bone structural units with a distribution of microcracks along cement lines, at the vicinity of lacunae, vascular pores or other defects. Since micro-cracks develop continuously with fatigue loading of the bone tissue, uniform distributions of yield strain and ultimate plastic strain up to the mean values observed in micro-pillar compression tests were assumed, which is in line with the argument made by Krajcinovic (19) in a damage model for bone under tension. 


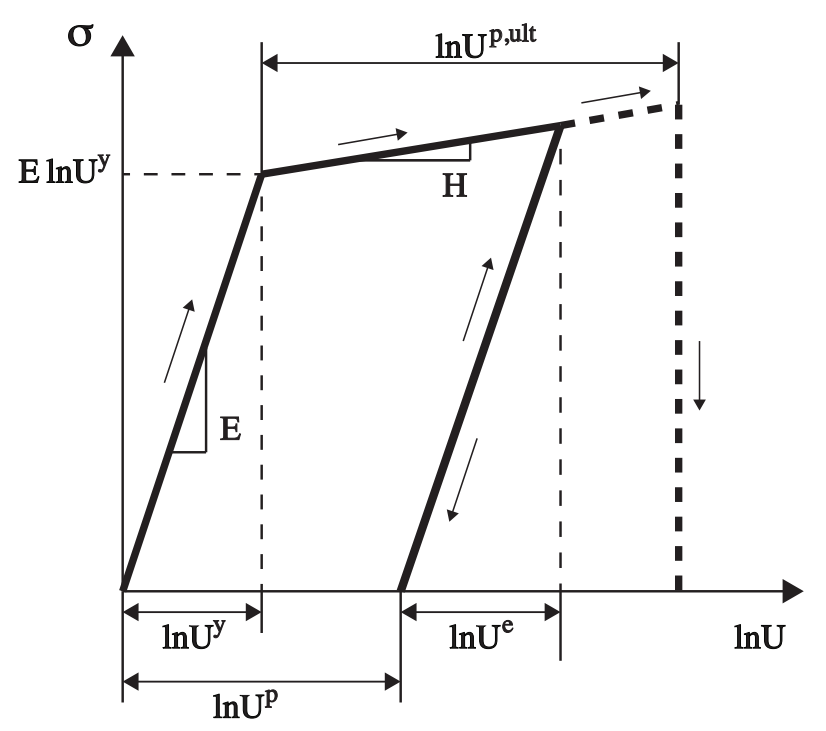

Supplementary Figure 9: Stress-strain behaviour: Elasto-plastic material response with linear hardening and subsequent failure.

Supplementary Table 1: Material constants of extracellular bone matrix.

\begin{tabular}{lcccc} 
& $E / \mathrm{GPa}$ & $H / \mathrm{GPa}$ & $\ln U^{y}$ & $\ln U^{p, u l t}$ \\
\hline Axial & $31.2 \pm 3.12$ & $0.0312 \pm 0.0031$ & $0.0241 \pm 0.0019$ & $0.08 \pm 0.008$ \\
Transv. & $16.5 \pm 1.65$ & $3.5 \pm 0.35$ & $0.0182 \pm 0.0015$ & $0.1 \pm 0.01$ \\
\hline
\end{tabular}
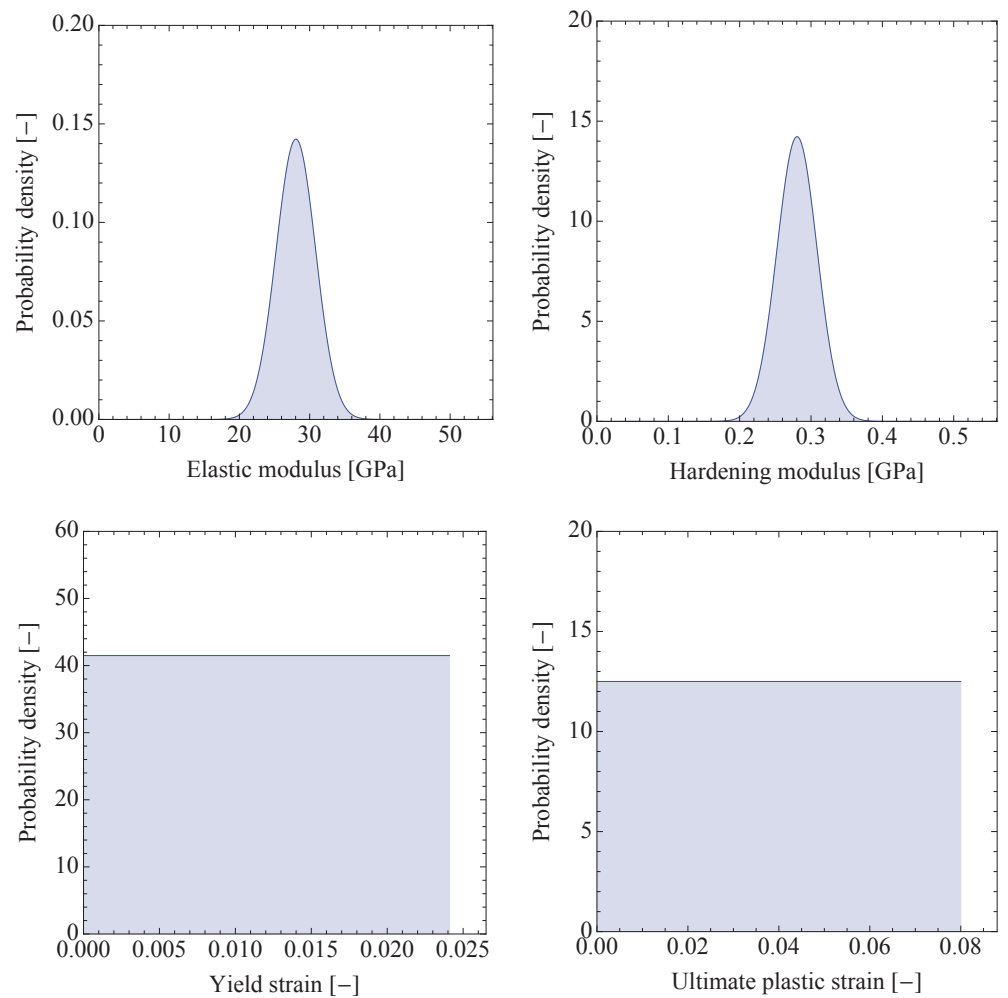

Supplementary Figure 10: Distributions of material properties for a macroscopic sample: Distributions of elastic modulus, hardening modulus, yield strain and ultimate plastic strain for the axial direction of bone structural units. 


\section{References}

[1] Pearce, A.I., Richards, R.G., Milz, S., Schneider, E. \& Pearce, S.G. Animal models for implant biomaterial research in bone: a review. Eur Cells Mater 13, 1 - 10 (2007).

[2] Ravaglioli, A. et al. Mineral evolution of bone. Biomaterials 17(6), 617 - 622 (1996).

[3] Wolfram, U., Wilke, H.-J. \& Zysset, P.K. Rehydration of vertebral trabecular bone: Influences on its anisotropy, its stiffness and the indentation work with a view to age, gender and vertebral level. Bone 46(2), 348 - 354 (2010).

[4] Morris, M.D. \& Mandair, G.S. Raman assessment of bone quality. Clin Orthop Relat R 469(8), 2160-2169 (2011).

[5] Akkus, O., Adar, F. \& Schaffler, M.B. Age-related changes in physicochemical properties of mineral crystals are related to impaired mechanical function of cortical bone. Bone 34(3), 443-453 (2004).

[6] Yerramshetty, J.S., Lind, C. \& Akkus, O. The compositional and physicochemical homogeneity of male femoral cortex increases after the sixth decade. Bone 39(6), 1236-1243 (2006).

[7] Oliver, W.C. \& Pharr, G.M. An improved technique for determining hardness and elastic modulus using load and displacement sensing indentation experiments. J Mater Res 7, 1564 - 1583 (1992).

[8] Reisinger, A.G., D.H. Pahr, D.H. \& Zysset, P.K. Principal stiffness orientation and degree of anisotropy of human osteons based on nanoindentation in three distinct planes. J Mech Behav Biomed 4(8), 2113 - 2127 (2011).

[9] Franzoso, G. \& Zysset, P.K. Elastic anisotropy of human cortical bone secondary osteons measured by nanoindentation. J Biomech Eng - T ASME 131(11), 117001 (2009).

[10] Spiesz, E.M., Roschger, P. \& Zysset, P.K. Elastic anisotropy of uniaxial mineralized collagen fibers measured using two-directional indentation. effects of hydration state and indentation depth. J Mech Behav Biomed 12, 20-28 (2012).

[11] Ziegler, J.F. \& Biersack, J.P. The Stopping and Range of Ions in Matter (Springer, New York, 1985).

[12] Michler, J., Wasmer, K., Meier, S., Ostlund, F. \& Leifer, K. Plastic deformation of gallium arsenide micropillars under uniaxial compression at room temperature. Appl Phys Lett 90(4), 043123 - 043123-3 (2007).

[13] Nalla, R. et al. Ultrastructural examination of dentin using focused ion-beam cross-sectioning and transmission electron microscopy. Micron 36, 672 - 680 (2005).

[14] Rabe, R. et al. Observation of fracture and plastic deformation during indentation and scratching inside the scanning electron microscope. Thin Solid Films 469, 206 - 213 (2004). 
[15] Zhang, H., Schuster, B.E., Wei, Q. \& Ramesh, K.T. The design of accurate micro-compression experiments. Scripta Mater 54(2), 181 - 186 (2006).

[16] Ashby, M. \& Jones, D. Engineering Materials (Pergamon Press, Oxford, 1980).

[17] R Development Core Team. R: A Language and Environment for Statistical Computing. (R Foundation for Statistical Computing, Vienna, 2008).

[18] Shapiro, S.S. \& Wilk, M.B. An analysis of variance test for normality (complete samples). Biometrika, 52(3/4), $591-611$ (1965).

[19] Krajcinovic, D., Trafimow, J. \& Sumarac, D. Simple constitutive model for a cortical bone. J Biomech 20(8), 779-784 (1987). 